\title{
An Actively Quenched Single Photon Detector with a Light Emitting Diode
}

\author{
David J. Starling ${ }^{1}$, Blake Burger ${ }^{2}$, Edward Miller ${ }^{2}$, Joseph Zolnowski ${ }^{2} \&$ Joseph Ranalli $^{2}$ \\ ${ }^{1}$ Division of Science, Pennsylvania State University, Hazleton, USA \\ ${ }^{2}$ College of Engineering, Pennsylvania State University, Hazleton, USA \\ Correspondence: David J. Starling, Penn State Hazleton, 76 University Drive, Hazleton, PA 18202, USA. Tel: \\ 1-570-450-3081. E-mail: starling@psu.edu
}

Received: September 10, 2015

Accepted: September 26, 2015 Online Published: December 18, 2015

doi:10.5539/mas.v10n1p114

URL: http://dx.doi.org/10.5539/mas.v10n1p114

This research is financed by Penn State Hazleton.

\begin{abstract}
Light emitting diodes (LEDs) have applications in many industries for illumination. However, the LED is not limited to the generation of light. In this article, we demonstrate and analyze a low-cost LED for use as a single photon detector, rather than as an emitter. While many technologies have been developed for single photon detection, the focus has been on timing resolution and efficiency-not on cost of production. We compare our device to commercial solutions and validate the efficacy of active quenching to improve maximum photon count rate. While this device may be useful for labs requiring a large number of low-cost detectors, it can also serve as a teaching tool in advanced laboratory settings.
\end{abstract}

Keywords: single photon detector, light emitting diode, active quenching, optics, electronics

\section{Introduction}

The detection of light is at the heart of many branches of science. In particular, single photon detection and counting are key parts of quantum optics and communication (Divochiy et al., 2008), astronomy (Nightingale, 1991), spectroscopy and general imaging (Tisa, Zappa, Tosi, \& Cova, 2007), to name a few. Therefore, it is no surprise that there currently exist a plethora of photon detection techniques.

Dating back to the nineteenth century, chemical photography played a crucial role in astronomy for many years, only to be replaced by the charge-coupled device (CCD) in the 1970s. The CCD is now the standard technology used in a variety of areas from amateur photography to scientific data collection. On the other hand, the photomultiplier tube (PMT) was developed in the 1930s to provide higher sensitivity and timing resolution (but no spatial resolution). The PMT is capable of multiplying a faint light signal into a measurable fountain of electrons. More recently, semiconductor materials have outpaced the PMT as the primary single photon detector technology. In particular, the avalanche photodiode (APD) is capable of detecting single photons at a high rate $(10 \mathrm{MHz})$ with very good timing accuracy $(100 \mathrm{ps})$ and very low noise $(<100 \mathrm{~Hz})$ (see, for example, Thorlabs model SPCM20A). Current state of the art systems have incorporated these APDs into 2-dimensional arrays capable of detecting single photon images with high timing resolution (Tisa et al., 2007). The APD has a number of drawbacks, however, including characteristically small detection areas and high bias voltage requirements. Modules capable of single photon detection, such as APDs and PMTs, are referred to collectively as single photon counting modules (SPCMs). In this article, we consider the use of the well-known light emitting diode (LED) as a possible alternative to the APD (Faramarzpour, Deen, Shirani, \& Fang, 2008; Niclass, Sergio, \& Charbon, 2006) to construct a low-cost SPCM.

The LED has grown in popularity dramatically due to its low energy requirements for lighting applications; in fact, the LED can even operate above unit efficiency by pumping heat from the environment into visible light (Santhanam, Gray, \& Ram, 2012). However, it is not the lighting capabilities of the LED that we wish to explore. In the May 2013 issue of "The Relaxation Times", a regular newsletter printed by TeachSpin, Inc., the author described the possible use of a LED as a photon detector, rather than a photon emitter (What's a SPAD?, 2013). From their experimental data, they demonstrated the linear response of the LED as well as its statistical behavior. 
In doing so, it was determined that the LED was behaving in a manner consistent with single photon detection, and even observed afterpulsing (i.e., a photon event triggering a second spurious avalanche of current), a common characteristic of APD devices (Liu, Hu, Campbell, Pan, \& Tashima, 2008).

Based upon their results, we believe the LED has the potential for useful scientific photon measurements, albeit with low detection efficiency. However, one common problem with diodes used for photon detection (including LEDs) is the slow reset time of the circuit due to their internal capacitance; this slow reset limits the maximum achievable count rate. A typical strategy to combat this limitation with APDs is the inclusion of an active reset or an active quenching circuit (Cova, Longoni, \& Ripamonti, 1982; Cova, Ghioni, Lacaita, Samori, \& Zappa, 1996; Gallivanoni, Rech, Resnati, Chioni, \& Cova, 2006; Zappa, Ghioni, Cova, Samori, \& Giudice, 2000). However, there has been no work on an actively quenched LED SPCM. In the following sections, we demonstrate passive and active quenching circuits using a LED for single photon detection.

\section{Method}

\subsection{Method Background: LED vs. APD}

Semiconductor photon detectors use common features of the diode along with the conversion of light energy to electrical current. A typical diode (e.g., Zener, APD, LED) is made up of two semiconductors placed in contact with one another; see, e.g., Boylestad and Nashelsky (1996) and Plonus (2001). One of the materials is an $n$-type semiconductor with electrons serving as the majority carrier. The other material is a $p$-type semiconductor with holes serving as the majority carrier. When these two materials are placed together, the electrons and holes near the junction combine in the depletion region. In this region, there are no majority carriers and so no substantial current can flow through the diode.

By applying a positive voltage to the $p$-type material (and negative to the $n$-type material), the holes and electrons will move to fill in the depletion region, thereby allowing current to flow. This is the usual configuration for an LED, where photons are created via electroluminescence; i.e., when electrons and holes combine in the depletion region, their energy is converted to photons. However, if a negative voltage is applied to the $p$-type material (and positive to the $n$-type material), the depletion region will only be further depleted of majority carriers and no current will flow. This is known as reverse bias and is the configuration used in photon detection.

When a diode is placed in reverse bias, there is a point at which stray electrons within the material (known as minority carriers) are accelerated to sufficient speeds by the electrical potential that they can knock valence electrons from their covalent bonds. This has an avalanche effect, knocking out more and more electrons and producing a large breakdown current. The voltage at which this occurs is known as the breakdown voltage or Zener potential $V_{Z}$. While the avalanche is typically caused by the always present minority carriers, in the case of the LED, the bias voltage $V_{b}$ is held below the Zener potential. In this case, the minority carriers do not have enough energy to cause an avalanche. However, an avalanche can be produced by an electron knocked free via the absorption of a single photon into the diode (i.e., the photoelectric effect). The high potential then accelerates the electron, potentially producing an avalanche as before.

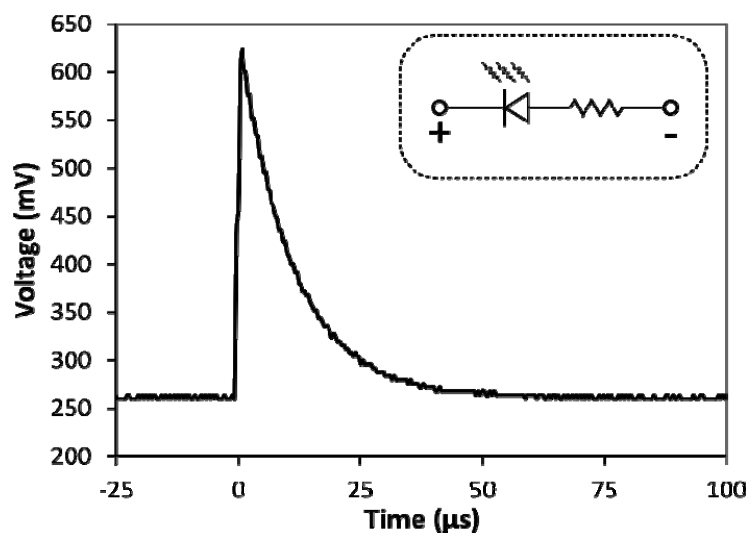

Figure 1. Passively quenched LED

A reverse-biased LED undergoing a photon-induced avalanche in the passively quenched configuration (shown 
in the inset). The exponential decay is due to the series resistance $R$ and internal capacitance $C$ of the LED (so $\tau=R C$ ). The $1 / e$ decay time is $\tau=11 \mu \mathrm{s}$ with an approximate series resistance of $100 \mathrm{k} \Omega$.

This process is possible due to the fact that an LED has the same basic structure as an APD, with a $p-n$ junction made of two semiconductors. Despite this similarity, there are a number of key differences. In particular, the APD has a very thick depletion region, improving photon absorption. In contrast, the LED has a very thin depletion region, resulting in better transparency and therefore good electroluminescence. However, the LED is capable of photon absorption, and it is this feature we wish to exploit.

\subsection{Passive Quenching}

If a LED is placed in reverse bias near its breakdown voltage and a photon is absorbed, current begins to flow. However, once the current begins to flow, the resistance of the circuit will cause a drop in the bias voltage via Ohm's Law. This drop in potential reduces the acceleration of the electrons in the semiconductor. This behavior passively quenches the flow of electrons, eventually reseting the diode back to its original non-conducting state. This passive quenching circuit creates a reduction in the bias voltage in an exponential fashion, shown Figure 1. By monitoring the potential across the resistor, the current in the circuit resulting from a photon-induced avalanche can be observed. The inset of the figure shows a circuit with the diode reverse-biased at approximately $24 \mathrm{~V}$ along with a resistor to quench the avalanche. We use an encapsulated gallium arsenide phosphide (GaAsP) LED (model 276-041) with a $5 \mathrm{~mm}$ lens, forward bias operating parameters of $2.6 \mathrm{~V}, 28 \mathrm{~mA}, 10$ mcd brightness and a peak wavelength of $650 \mathrm{~nm}$.

We test the same LED using a variety of resistors between $11 \mathrm{k} \Omega$ and $100 \mathrm{k} \Omega$. The decay time can be found using a best fit curve of the form $e^{-t / \tau}$ and the Pearson's $\chi^{2}$ test as the fit parameter. We plot the decay time $\tau$ as a function of resistance and find a linear relationship as shown in Figure 2.

The minimum reverse bias voltage value found to produce avalanching behavior was $V_{b}=22.2 \mathrm{~V}$. By applying more voltage in excess of $V_{b}$ (see $V_{e x}$ in Figure 3), the probability of an absorbed photon creating an avalanche increases. However, this also produces an increased number of dark counts, the most common noise source for SPCMs. These unavoidable counts are caused by thermal excitation or minority carriers and add to photon detection events. However, they can be reduced in typical SPCMs using temperature control. We covered the detector with an opaque shroud to measure dark counts, with results showin in Figure 3. In order to count the photon detection events, the voltage output from the LED was passed to an ultrafast fast comparator (model AD8561) to create square TTL pulses; the resulting pulses were sent to a custom FPGA pulse counter (NIST Physical Measurement Laboratory, 2014).

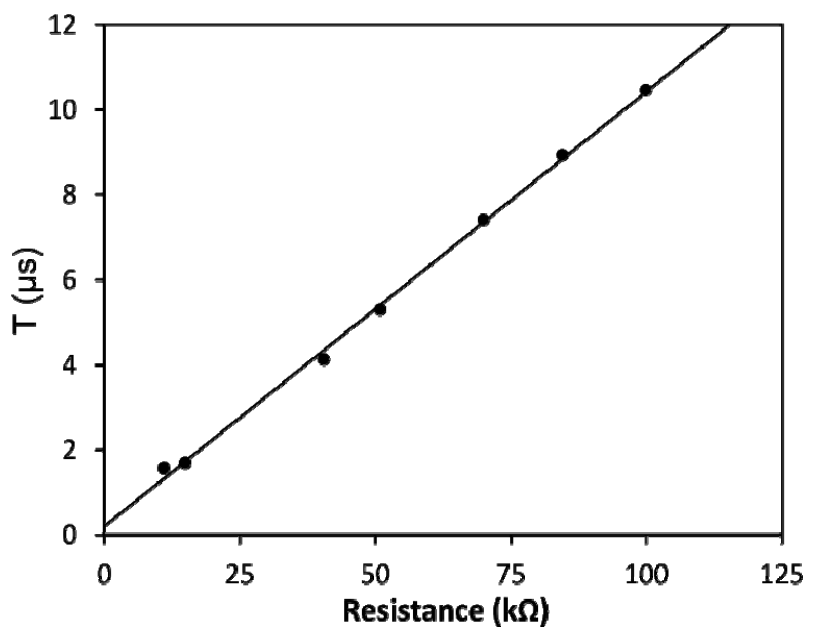

Figure 2. Reset time of LED SPCM

The decay time of a passively quenched LED at room temperature is shown as a function of the series resistance of the circuit. The relationship is approximately linear with a slope of $0.102 \pm 0.002 \mu \mathrm{s} / \mathrm{k} \Omega$. This implies that the capacitance of the LED is about $C=0.102 \mathrm{nF}$. 


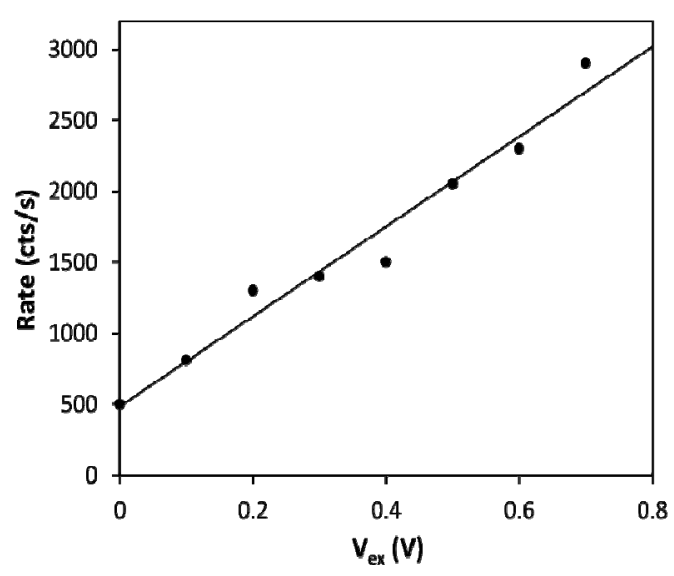

Figure 3. Dark counts of LED SPCM

The dark counts at room temperature for a passively quenched LED with an $81 \mathrm{k} \Omega$ resistor are shown as a function of the applied excess bias voltage. The relationship is approximately linear with a slope of $3170 \pm 240$ $\mathrm{Hz} / \mathrm{V}$.

\subsection{Active Quenching}

While the passively quenched circuit offers a simple solution to measure single photons, it is possible to expand this analysis to more modern designs. State of the art semiconductor SPCMs have an active circuit that quickly lowers the bias voltage below the Zener potential of the APD. When this technique is applied to the LED SPCM, the drop in voltage prematurely ends the avalanche in a time significantly shorter than the passive decay time. Once the avalanche has ended, the bias voltage is returned to its original value to prepare the LED for another photon detection event. A side effect of this process is that the maximum possible bias voltage applied to the LED is increased, resulting in more efficient detection than in the passively quenched design.

In our design, the ultrafast comparator ( $\mathrm{C}$ in Figure 4) switches on when a photon is detected. This signal triggers a metal-oxide-semiconductor field-effect transistor (MOSFET, $\mathrm{T}$ in Figure 4, model IRF510) to enter active mode, draining the source current through the $920 \Omega$ resistor to ground and consequently lowering the bias voltage applied to the LED. Once the voltage across the LED drops below a reference value, set by $V_{C}$, the comparator switches off and the MOSFET enters cutoff mode, thereby resetting the bias voltage to its original value. The resulting avalanche current does not decay exponentially but is instead truncated in a time determined by the response of the comparator and transitor. With this actively controlled circuit, we measured the maximum count rate as a proxy for the exponential reset time.

Finally, we characterized the response of the actively quenched LED SPCM to incident light. To do so, we measured the photon count rate and incident power produced by a HeNe laser, attenuated using rotated polarizers, as shown in Figure 4. The response is shown in Figure 5, and the results will be discussed in the following section.

\section{Results}

\subsection{Passive Quenching}

We evaluated a standard low-cost LED for use as a SPCM in two configurations. In the passive configuration, we found that the LED has the characteristic behavior associated with photon avalanche events, including exponential current decay, resistance-dependent reset time and dark counts that vary linearly with excess bias voltage. In particular, it was possible to reduce the reset time to $1.56 \mu$ s with an $11 \mathrm{k} \Omega$ resistor. This decay time sets the limit on the possible count rate $R$ for the passively quenched configuration, given by $R=1 / \tau=$ $640 \mathrm{kHz}$ (compare to modern APD SPCMs with count rates above $10 \mathrm{MHz}$ ). This disadvantage can be partially overcome by employing active quenching.

On the other hand, the minimum dark count rate was measured for the LED with an $81 \mathrm{k} \Omega$ resistor to be about $500 \mathrm{~Hz}$ at room temperature. This value is comparable to many cooled APD SPCMs. However, at this large resistance, the maximum possible count rate was limited. 


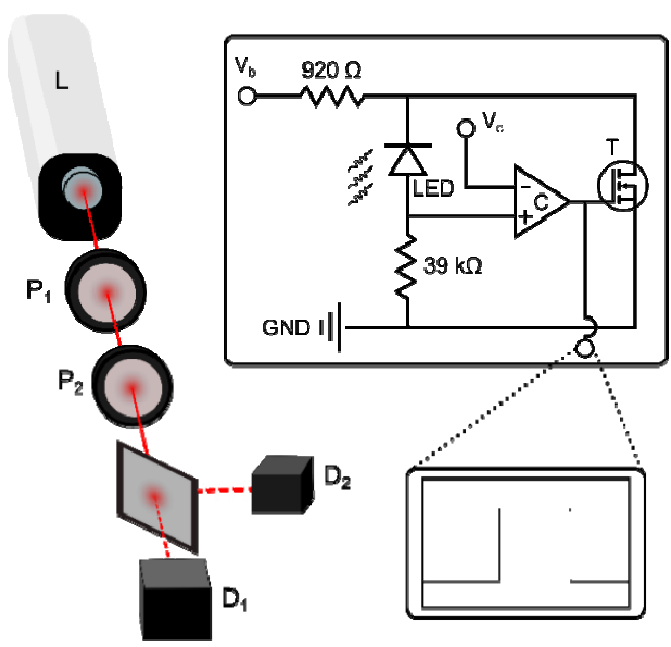

Figure 4. Active quenching experimental setup

The circuit diagram for the active quenching configuration (right) along with the experimental setup to measure the response (left) are shown. In the circuit, $V_{b}$ is the bias voltage applied to the LED, and $V_{c}$ is the comparison voltage used to trigger the comparator $(\mathrm{C})$. The comparator produces a square pulse when the LED avalanche occurs; this square pulse switches the MOSFET from cutoff mode to active mode, allowing the bias voltage to drain to ground through the $920 \Omega$ resistor, reducing the voltage across the LED. Once the bias voltage drops (stopping the current through the LED), the comparator turns off so that the LED bias returns to $V_{b}$ and the device is ready to detect another photon. In the optical setup, the beam from a HeNe laser (L) passes through two rotation-mounted polarizers $P_{1}$ and $P_{2}$. The power is adjusted using the polarizers and then measured with the LED SPCM $D_{1}$ and a calibrated power meter $D_{2}$ for comparison.

\subsection{Active Quenching}

The actively quenched circuit was capable of measuring significantly higher count rates using the same resistor and LED combination when compared to the passive design. In particular, the LED with a $39 \mathrm{k} \Omega$ resistor had a maximum count rate of $10 \mathrm{kHz}$ in the passive configuration, but $130 \mathrm{kHz}$ in the active configuration (under identical lighting conditions at room temperature). This behavior can be explained in part by the higher bias voltages possible in the active configuration (before the LED ceases to avalanche). Additionally, the active quenching protects the LED from overheating by limiting the average current through the diode.

While the reset behavior (delay and length) of the active circuit can be adjusted using faster electronics and variable capacitors, we found that a straightforward design was sufficient to vastly increase the maximum possible count rate. We obtained an increase in maximum count rate by a factor of 10 or more for the active quenching circuit when compared to passive quenching. Therefore, with suitable electronics, a rate of $6.4 \mathrm{MHz}$ (a factor of 10 higher than the passive $640 \mathrm{kHz}$ detector we tested) is expected. This rate is comparable to modern commercial APDs.

Using the actively quenched LED SPCM, we next tested its response to incoming light. From Figure 5, we see that the detector responds nonlinearly. In particular, counts respond linearly in two regimes: below $20 \mu \mathrm{W}$ and above $100 \mu \mathrm{W}$, with a nonlinear transition between. This is likely the result of electronic delay or the long rise time of the transistor since this behavior was not exhibited in the passive design. Also, note that the incident power includes all spatial modes of the laser (captured by the calibrated power meter), while the LED only captures a small fraction of these modes due to its size and orientation. For this reason, it is difficult to approximate the efficiency of the SPCM.

Despite the difficulty in determining the efficiency of detection, it is clear that the efficiency of the LED is significantly lower than that of a traditional APD, most likely due to its small depletion region-both in thickness and in cross-section. However, commercially available LEDs with large cross-sections should alleviate difficulties with light coupling. On the other hand, if one were to fabricate an LED with a thicker depletion region, then the required bias voltage would increase and more specialized, high voltage equipment would be required (as is the case with an APD SPCM). 


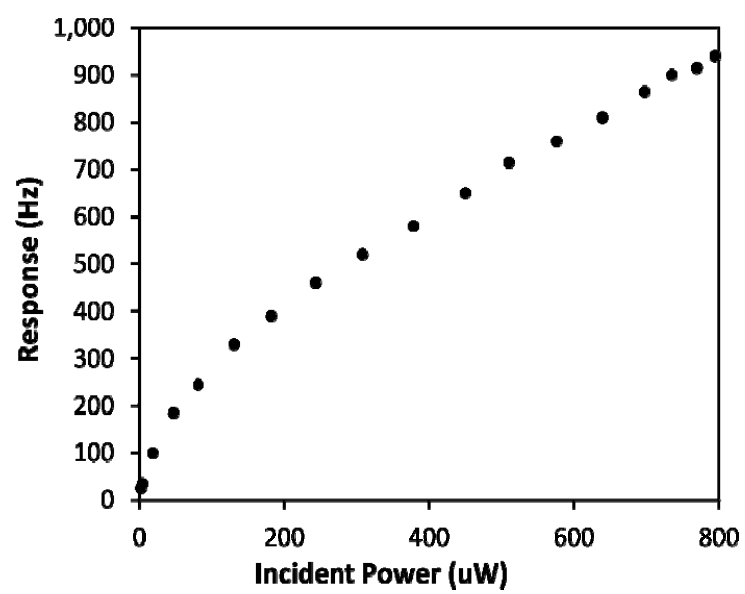

Figure 5. Response of Actively Quenched SPCM

The count rate response of the actively quenched LED at room temperature is shown as a function of incident power. Dark counts have been subtracted in this figure.

\section{Discussion}

We first explored the use of a LED as a SPCM in a passive circuit. This passive design introduced a variety of key considerations for detectors: max count rate, dark counts, afterpulsing, bias voltage, internal capacitance and response. We found that a LED can be used effectively as a SPCM, but was limited to sub megahertz count rates. From there, we expanded on this simple design to improve the maximum count rate. We found a factor of 10 improvement was possible using a straightforward active quenching design. Therefore, we expect a detection rate exceeding $5 \mathrm{MHz}$ is possible with a low-cost LED.

Using a LED as a SPCM has two key advantages in relation to APDs: they are inexpensive and plentiful, and they can be used with standard and inexpensive electronic hardware at low voltages (less than $30 \mathrm{~V}$ ). APDs, on the other hand must be ordered or fabricated, often resulting in longer procurement times. Furthermore, most silicon APDs require more than $100 \mathrm{~V}$ (often over $200 \mathrm{~V}$ ) to produce avalanching behavior and are equipped with small solder points which add to experimental difficulty. Additionally, APDs tend to have very small active regions, requiring high-numerical-aperture optics and careful alignment; conversely, large-area LEDs are common and affordable. For these reasons, the LED is a promising solution for research labs looking to employ multiple SPCMs at low cost.

However, the detection efficiency of the LED SPCM is low enough that it is unlikely to find use in experiments requiring high efficiency, such as squeezing. However, with a large aperture LED and fast electronics, a LED SPCM could be used for multimode detection with good timing resolution (Howland, Lum, Ware, \& Howell, 2013), shot noise limited power measurements or simply as an experimental tool for statistical analysis (Soares, Mendonca, \& Ramos, 2014).

It should be noted that the parameter space for experimentation and analysis is quite large. In this work, we focused on improving the maximum count rate for practical applications. However, further study may include a better characterization of the quantum efficiency of LEDs, active cooling to reduce dark counts or fine-tuning of the electronic components to reduce nonlinearities.

\section{Acknowledgments}

We gratefully acknowledge the support from Penn State Hazleton and the College of Engineering, as well as work conducted by Janak Jethva, Ryan Kanick, Thomas Klein and Richard Michael on an earlier version of these experiments.

\section{References}

Boylestad, R. L., \& Nashelsky, L. (1996). Electronic devices and circuit theory (6th ed.). Columbus, OH: Prentice Hall.

Cova, S., Ghioni, M., Lacaita, A., Samori, C., \& Zappa, F. (1996). Avalanche Photodiodes and Quenching Circuits for Single-photon Detection. Appl. Opt., 35(12), 1956-1976. http://dx.doi.org/10.1364/AO.35.001956 
Cova, S., Longoni, A., \& Ripamonti, G. (1982). Active-Quenching and Gating Circuits for Single-Photon Avalanche Diodes (SPADs). IEEE Trans. on Nucl. Sci., 29(1), 599-601. http://dx.doi.org/10.1109/TNS.1982.4335917

Divochiy, A., Marsili, F., Bitauld, D, Gaggero, A., Leoni, R., Mattioli, F., \& Fiore, A. (2008). Superconducting Nanowire Photon-number-resolving Detector at Telecommunication Wavelengths. Nature Photonics, 2, 302-306. http://dx.doi.org/10.1038/nphoton.2008.51

Faramarzpour, N., Deen, M. J., Shirani, S., \& Fang, Q. (2008). Fully Integrated Single Photon Avalanche Diode Detector in Standard CMOS 0.18- $\mu \mathrm{m}$ Technology. IEEE Trans. on Elec. Dev., 55(3), 760-767. http://dx.doi.org/10.1109/TED.2007.914839

Gallivanoni, A., Rech, I., Resnati, D., Ghioni, M., \& Cova, S. (2006). Monolithic Active Quenching and Picosecond Timing Circuit Suitable for Large-area Single-photon Avalanche Diodes. Opt. Exp., 14(12), 5021-5030. http://dx.doi.org/10.1364/OE.14.005021

Howland, G. A., Lum, D. J., Ware, M. R., \& Howell, J. C. (2013). Photon Counting Compressive Depth Mapping. Optics Express, 21(20), 23822-23837. http://dx.doi.org/10.1364/OE.21.023822

Liu, M., Hu, C., Campbell, J. C., Pan, Z., \& Tashima, M. M. (2008). Reduce Afterpulsing of Single Photon Avalanche Diodes Using Passive Quenching With Active Reset. IEEE J. of Quant. Elec., 44(5), 430-434. http://dx.doi.org/10.1109/JQE.2007.916688

Niclass, C., Sergio, M., \& Charbon, E. (2006). A Single Photon Avalanche Diode Array Fabricated in $0.35 \mu \mathrm{m}$ CMOS and based on an Event-Driven Readout for TCSPC Experiments. Adv. Photon Counting Techniques 6372, 63720S-12. http://dx.doi.org/10.1117/12.685974

Nightingale, N. S. (1991). A New Silicon Avalanche Photodiode Photon Counting Detector Module for Astronomy. Exp. Astro., 1(6), 407-422. http://dx.doi.org/10.1007/BF00426721

NIST Physical Measurement Laboratory. (2014). FPGA-based multicoincidence recipe and software. Retrieved from http://www.nist.gov/pml/div684/grp03/multicoincidence.cfm

Plonus, M. (2001). Electronics and communications for scientists and engineers (1st ed.). New York, NY: Harcourt/Academic Press.

Santhanam, P., Gray, Jr., D. J., \& Ram, R. J. (2012). Thermoelectrically Pumped Light-Emitting Diodes Operating above Unity Efficiency. Phys. Rev. Lett., $108(9), \quad 097403$. http://dx.doi.org/10.1103/PhysRevLett.108.097403

Soares, E. J. L., Mendonca, F. A., \& Ramos, R. V. (2014). Quantum Random Number Generator Using Only One Single-Photon Detector. Photonics Technology Letters, IEEE, 26(9), 851-853. http://dx.doi.org/10.1109/LPT.2014.2302436

The Relaxation Times. (2013). What's a SPAD? Retrieved from http://www.teachspin.com/newsletters/ TeachSpin_MAY13FINALFOR\%20WEB.pdf

Tisa, S., Zappa, F., Tosi, A., \& Cova, S. (2007). Electronics for Single Photon Avalanche Diode Arrays. Sensors and Actuators A, 140(1), 113-122. http://dx.doi.org/10.1016/j.sna.2007.06.022

Zappa, F., Ghioni, M., Cova, S., Samori, C., \& Giudice, A. C. (2000). An Integrated Active-Quenching Circuit for Single-Photon Avalanche Diodes. IEEE Trans. on Intru. and Meas., 49(6), 1167-1175. http://dx.doi.org/10.1109/19.893251

\section{Copyrights}

Copyright for this article is retained by the author(s), with first publication rights granted to the journal.

This is an open-access article distributed under the terms and conditions of the Creative Commons Attribution license (http://creativecommons.org/licenses/by/3.0/). 